\title{
ON SERRE MICROFIBRATIONS AND A LEMMA OF M. WEISS
}

\author{
GEORGE RAPTIS \\ Universität Regensburg, Fakultät für Mathematik, \\ 93040 Regensburg, Germany \\ e-mail: georgios.raptis@ur.de
}

(Received 21 April 2016; revised 14 September 2016; accepted 24 September 2016; first published online 20 March 2017)

\begin{abstract}
We show that every fibrewise map from a Serre microfibration to a Serre fibration is $n$-connected if it is fibrewise $n$-connected. This generalises a result of $\mathrm{M}$. Weiss and related results by Bökstedt-Madsen and Galatius-Randal-Williams. We also discuss an application to configuration spaces.
\end{abstract}

2000 Mathematics Subject Classification. 55R65, 55R70, 55R80.

1. Introduction. How can one determine the homotopy types of the homotopy fibres of a map $f: X \rightarrow Y$ from the homotopy types of its strict fibres? These are the same in the case where $f$ is a (quasi-)fibration, but the comparison between the two types of fibres is a difficult task in general. Even in the case where all strict fibres are homotopy equivalent, more information is required in order to identify this common homotopy type with that of the homotopy fibre. The required information should provide a way of understanding how the individual fibres are organised collectively in a parameterised family of spaces. This information may be expressed by different types of properties: in terms of lifting properties, as in the case of Serre/Hurewicz fibrations, in terms of properties of $f$ locally in $Y$, as in the case of local quasi-fibrations and related notions, or in terms of point-set topological assumptions on $X, Y$ and $f$ as, for example, in the case of results about cell-like maps.

In this note, we will be interested in the following notion from [10] which states a weak lifting property.

Definition 1.1. A map $p: E \rightarrow B$ is called a Serre microfibration if for any $k \geq 0$ and any commutative diagram

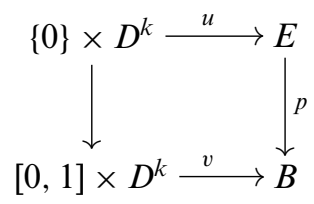

there exists an $\epsilon>0$ and a map $h:[0, \epsilon] \times D^{k} \rightarrow E$ such that $h(0, x)=u(x)$ and $p \circ h(t, x)=v(t, x)$ for all $x \in D^{k}$ and $t \in[0, \epsilon]$.

ExAmPLE 1.2. Let $q: V \rightarrow B$ be a Serre fibration and $E \subset V$ an open subset. Then, $q_{\mid E}: E \rightarrow B$ is a Serre microfibration. More generally, an open subspace of the total space of a Serre microfibration defines again a Serre microfibration. 
Weiss' microfibration lemma [10, Lemma 2.2] states that a Serre microfibration $p$ : $E \rightarrow B$ with weakly contractible fibres is actually a Serre fibration. As a consequence, it is also a weak homotopy equivalence. This result was generalised in [3] as follows: if the fibres of $p$ are $n$-connected, then $p$ has the right lifting property with respect to the inclusions

$$
\{0\} \times D^{k} \rightarrow[0,1] \times D^{k}, k \leq n .
$$

As a consequence, each map from a fibre to the homotopy fibre is $n$-connected, hence the homotopy fibres are $n$-connected, and the map $p$ is $(n+1)$-connected [3, Proposition 2.6].

The question of extending these results to arbitrary Serre microfibrations, whose fibres are abstractly weakly (or $n$-) homotopy equivalent, faces the problem of finding a uniform way of comparing them. In this direction, Bökstedt and Madsen [1, Lemma 3.6] proved, under certain point-set topological assumptions, that given a commutative diagram as follows, where the top map is an open inclusion and $p$ is the projection,

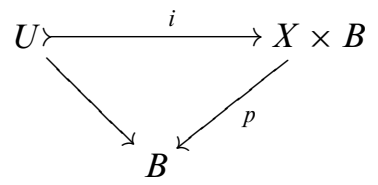

then $i$ is a weak homotopy equivalence if for every $b \in B$, the map on fibres, $U \cap p^{-1}(b) \rightarrow X$, is a weak homotopy equivalence.

The statement below generalises these results to the case of fibrewise $n$-connected maps with target an arbitrary fibration. We say that a map is a Serre $n$-fibration if it has the right lifting property with respect to the inclusions in $\left(*_{\leq n}\right)$ above.

THEOREM 1.3. Let $p: E \rightarrow B$ be a Serre microfibration, $q: V \rightarrow B$ be a Serre fibration and $f: E \rightarrow V$ a map over $B$. Suppose that $f_{b}: p^{-1}(b) \rightarrow q^{-1}(b)$ is $(n+1)$ connected, $n \geq 0$, for all $b \in B$. Then, $p$ is a Serre $n$-fibration and, as a consequence, the map $f: E \rightarrow V$ is $(n+1)$-connected.

REMARK 1.4. The statement is true, of course, for arbitrary maps $q: V \rightarrow B$ if we replace the fibres of $q$ with the homotopy fibres.

The proof of Theorem 1.3 will be given in Section 2. In Section 3, we give an application to configuration spaces and discuss the connection with the context of abstract transversality that was used in [1] and [3].

2. The Proof of Theorem 1.3. The proof of Theorem 1.3 follows [10, Lemma 2.2] and [3, Proposition 2.6].

Let $I=[0,1]$ be the unit interval. Let $X^{I}=\operatorname{Map}(I, X)$ be the space of paths with the compact-open topology. The following observation is due to Weiss [10, Lemma 2.2].

Lemma 2.1. Let $p: E \rightarrow B$ be a Serre microfibration. Then, $p^{I}: E^{I} \rightarrow B^{I}$ is also a Serre microfibration.

Proof. This follows directly by adjunction.

We also recall the following lemma from [3, p. 8]. 
LEMMA 2.2 (Galatius-Randal-Williams). Let $(Y, X)$ be a finite $C W$ pair and $p$ : $E \rightarrow B$ a Serre microfibration. Suppose we are given a lifting problem

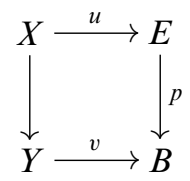

If there exists a map $h: Y \rightarrow E$ lifting $v$ and such that $h_{\mid X}$ is fibrewise homotopic to $u$, then there is also a map $h^{\prime}: Y \rightarrow E$ that makes the diagram commute strictly.

Lemma 2.3. Let $p, q$ and $f$ be as in Theorem 1.3. Then, the map

$$
\left(f^{I}\right)_{b}:\left(p^{I}\right)^{-1}(b) \rightarrow\left(q^{I}\right)^{-1}(b)
$$

is n-connected for all $b: I \rightarrow B$.

Proof. By adjunction, it suffices to prove that for each $k \leq n$ and each lifting problem

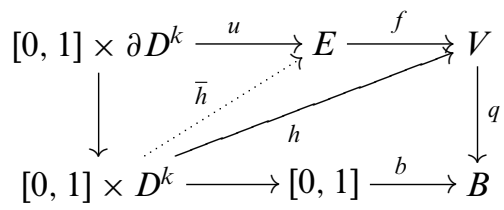

there exists a map $\bar{h}:[0,1] \times D^{k} \rightarrow E$ over $B$ so that the upper triangle commutes, and the triangle in the middle commutes up to fibrewise homotopy over $B$ and relative to $[0,1] \times \partial D^{k}$. For $t \in[0,1]$, consider the restricted diagram

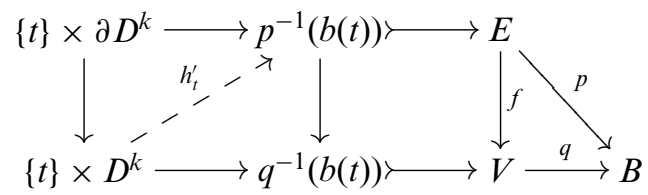

where the lower composite is the constant map at $b(t) \in B$. By assumption, there exists a map $h_{t}^{\prime}:\{t\} \times D^{k} \rightarrow p^{-1}(b(t))$ such that the upper triangle commutes and the lower triangle commutes up to homotopy relative to $\partial D^{k}$. The microfibration property of $p$ applied to the commutative diagram

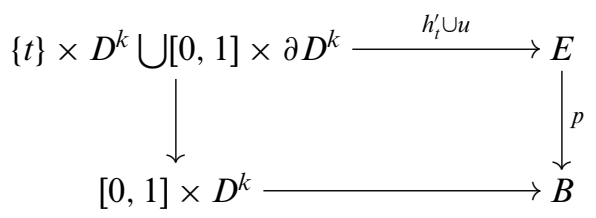


provides an extension of the lift $h_{t}^{\prime}:\{t\} \times D^{k} \rightarrow p^{-1}(b(t)) \subseteq E$ to a map $h_{t, \epsilon}^{\prime}$ defined on $[t-\epsilon(t), t+\epsilon(t)] \times D^{k}$, for some $\epsilon(t)>0$, and such that the diagram commutes

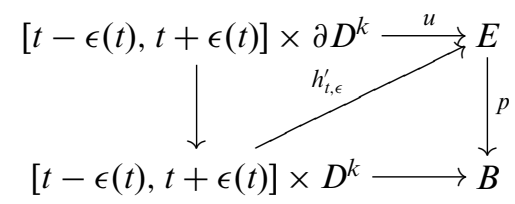

Moreover, since the map $f \circ h_{t}^{\prime}:\{t\} \times D^{k} \rightarrow V$ is fibrewise homotopic to the restriction $h_{\left\{\{t\} \times D^{k}\right.}$ relative to $\{t\} \times \partial D^{k}$, it follows that for each $s \in[t-\epsilon(t), t+\epsilon(t)]$, the map

$$
\{s\} \times D^{k} \subseteq[t-\epsilon(t), t+\epsilon(t)] \times D^{k} \stackrel{h_{t, \epsilon}^{\prime}}{\rightarrow} E \stackrel{f}{\rightarrow} V
$$

is fibrewise homotopic to the corresponding restriction of $h$ relative to $\{s\} \times \partial D^{k}$. This uses that $q$ is a Serre fibration.

By the Lebesgue lemma, there is $N>0$ and maps

$$
h_{i}^{\prime}:[i / N,(i+1) / N] \times D^{k} \rightarrow E,
$$

for $i=0, \ldots, N-1$, such that:

(i) $h_{i}^{\prime}$ and $h_{i+1}^{\prime}$ agree on $\{(i+1) / N\} \times \partial D^{k}$,

(ii) $p \circ h_{i}^{\prime}$ agrees with $b$ on $[i / N,(i+1) / N]$,

(iii) the restrictions of $f \circ h_{i}^{\prime}$ and $f \circ h_{i+1}^{\prime}$ to $\{(i+1) / N\} \times D^{k}$ are homotopic relative to $\{(i+1) / N\} \times \partial D^{k}$, by a fibrewise homotopy over $B$, to the restriction of $h$.

The connectivity assumption and (iii) imply that the restrictions of

$$
h_{i}^{\prime}, h_{i+1}^{\prime}:\{(i+1) / N\} \times D^{k} \rightarrow p^{-1}\left(b\left(\frac{i+1}{N}\right)\right)
$$

are homotopic relative $\{(i+1) / N\} \times \partial D^{k}$. Thus, inductively, we obtain diagrams as follows, $i \geq 0$,

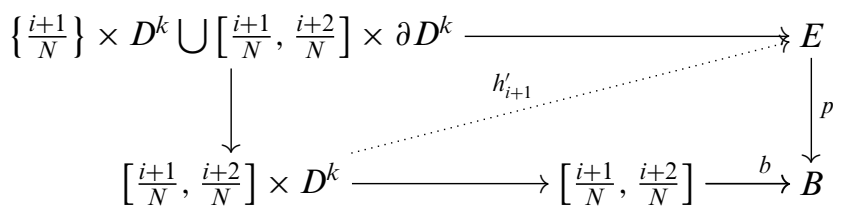

where the top map is defined by $h_{i}^{\prime}$ and $u$ and the upper triangle commutes up to fibrewise homotopy. Then, Lemma 2.2 shows that there is a map

$$
\bar{h}_{i+1}:[(i+1) / N,(i+2) / N] \times D^{k} \rightarrow E
$$

so that the diagram commutes strictly. This way, we inductively adjust the lifts $h_{i}^{\prime}$ so that they agree at the endpoints and obtain an extension of $u$,

$$
\bar{h}:[0,1] \times D^{k} \rightarrow E .
$$


The map $f \circ \bar{h}$ is then homotopic to $h$ fibrewise over $B$ and relative to $[0,1] \times \partial D^{k}$ because their restrictions to $\{0\} \times D^{k}$ are homotopic, by construction, and $q$ is a Serre fibration.

Proof of Theorem 1.3. We show that every diagram

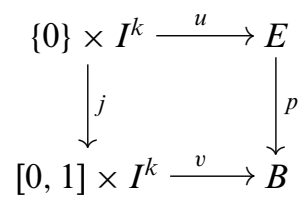

admits a diagonal filler for all $k \leq n$.

Since $q: V \rightarrow B$ is a Serre fibration, there is a map $g$ such that the following diagram commutes:

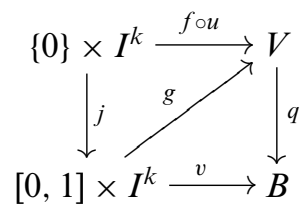

By Lemma 2.3, the map

$$
\left(f^{I^{k+1}}\right)_{v}:\left(p^{I^{k+1}}\right)^{-1}(v) \rightarrow\left(q^{I^{k+1}}\right)^{-1}(v)
$$

is 0 -connected for $k \leq n$. Therefore, there is a map $h:[0,1] \times I^{k} \rightarrow E$ so that the lower triangle of (1) commutes and $f \circ h$ belongs to the same path component of $\left(q^{I^{k+1}}\right)^{-1}(v)$ as $g$ does.

We claim that $u$ and $h \circ j$ are fibrewise homotopic over B. By Lemma 2.3, the map

$$
\left(f^{I^{k}}\right)_{v \circ j}:\left(p^{I^{k}}\right)^{-1}(v \circ j) \rightarrow\left(q^{I^{k}}\right)^{-1}(v \circ j)
$$

is 1-connected for $k \leq n$. The maps $f \circ u$ and $f \circ(h \circ j)$ are in the same path component of $\left(q^{I^{k}}\right)^{-1}(v \circ j)$ and hence so are the maps $u$ and $h \circ j$. Therefore, using Lemma 2.2, it follows that a diagonal filler to the lifting problem (1) exists, hence $p$ is a Serre $n$-fibration.

Since $p$ is a Serre $n$-fibration, the map $p^{-1}(b) \rightarrow \operatorname{hofib}_{b}(p)$ is $n$-connected. Then, the lower map between homotopy fibres in the diagram,

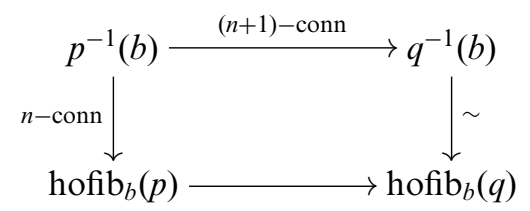

is $(n+1)$-connected. By the long exact sequence of homotopy groups, it follows that $f$ is $(n+1)$-connected.

REMARK 2.4. It would be interesting to investigate partial converses of Theorem 1.3. The problem is as follows: given a Serre microfibration $p: E \rightarrow B$, a Serre fibration $q: V \rightarrow B$ and a map $f: E \rightarrow V$ over $B$ which is a weak homotopy (or $n$-)equivalence, 
when are the maps $f_{b}: p^{-1}(b) \rightarrow q^{-1}(b)$ also weak homotopy equivalences? If $p$ is a Serre $n$-fibration and $f$ is $n$-connected, then it is easy to see that $f_{b}$ is $n$-connected for all $b \in B$, however, the converse claim is not true in general.

\section{Applications.}

3.1. Relative configuration spaces. In [8], we considered configuration spaces associated with maps as an attempt to deal with the lack of functoriality. We recall that given a space $X$, the space of (ordered) configurations of $n$ points in $X$ is defined to be the subspace $F_{n}(X) \subset X^{n}$ consisting of the $n$-tuples of pairwise distinct points. If $X$ is a topological manifold, then it is well known that the projection onto the first $n$ coordinates, $\pi_{n}: F_{n+1}(X) \rightarrow F_{n}(X)$, is a fibre bundle.

Given a map $f: X \rightarrow Y$, the relative (ordered) configuration space of $n$ points $F_{n}(f)$ is the subspace of $F_{n}(X)$ which consists of the configurations in $X$ whose images in $Y$ are $n$-tuples of pairwise distinct points. There is an obvious zigzag of maps

$$
F_{n}(X) \hookleftarrow F_{n}(f) \stackrel{f_{*}}{\rightarrow} F_{n}(Y) .
$$

If $Y$ is a Hausdorff space, then $F_{n}(f) \subset F_{n}(X)$ is open. If in addition $X$ is a topological manifold, then the projection

$$
F_{n+1}(f) \rightarrow F_{n}(f)
$$

is a Serre microfibration. Indeed, $F_{n+1}(f)$ is then an open subspace of the following pullback fibration:

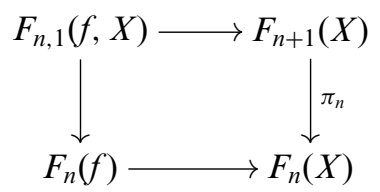

We recall the definition of cellular and cell-like maps, see, e.g., [4]. A compact subset $K \subset M$ of a topological $d$-manifold is cellular if there are $d$-dimensional balls $B_{i} \subset M, i \geq 1$, with $B_{i+1} \subset \operatorname{int}\left(B_{i}\right)$ and

$$
K=\bigcap_{i=1}^{\infty} B_{i} .
$$

It follows that, for $x \in K$, the spaces $M-\{x\}$ and $M-K$ are homeomorphic (see [2]) and the inclusion

$$
M-K \longrightarrow M-\{x\}
$$

is a homotopy equivalence. A map $f: M \rightarrow Y$ is cellular if $f^{-1}(y) \subset M$ is cellular for all $y \in Y$. A space $K$ is cell-like if there is a topological manifold $M$ and an embedding $\iota: K \hookrightarrow M$ such that $\iota(K)$ is cellular in $M$ [4]. There exist embeddings of cell-like spaces that are not cellular, that is, cellularity depends on the embedding - see [6] for a general cellularity criterion. For finite-dimensional compact metric spaces, being cell-like is equivalent to having trivial shape [5]. A map $f: X \rightarrow Y$ is cell-like if $f^{-1}(y)$ is cell-like 
for all $y \in Y$. Proper cell-like maps between ENRs are hereditary proper homotopy equivalences [4, Theorem 1.2].

We obtain the following result which generalises [8, Theorem 4.5].

Proposition 3.1. Let $M$ be a topological d-manifold (without boundary), $Y$ an ENR and $f: M \rightarrow Y$ a proper cellular map. Then, the maps $F_{n}(f) \rightarrow F_{n}(M)$ and $f_{*}:$ $F_{n}(f) \rightarrow F_{n}(Y)$ are homotopy equivalences for all $n \geq 1$.

Proof. We show inductively that $F_{n}(f) \rightarrow F_{n}(M)$ is a weak homotopy equivalence. Consider the following diagram:

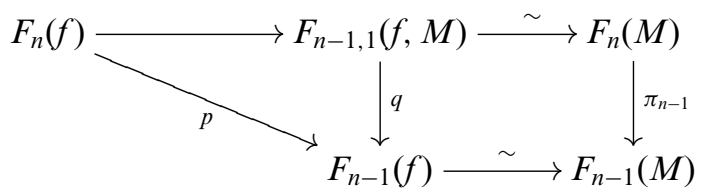

where the square on the right is a pullback by definition. We know that the projection $p$ is a Serre microfibration from the discussion above. For any $\underline{m}=\left(m_{1}, \ldots, m_{n-1}\right) \in$ $F_{n-1}(f)$, the map $p^{-1}(\underline{m}) \rightarrow q^{-1}(m)$ is given by the inclusion

$$
\left(M-f^{-1}\left(f\left(\left\{m_{1}, \ldots, m_{n-1}\right\}\right)\right)\right) \rightarrow\left(M-\left\{m_{1}, \ldots, m_{n-1}\right\}\right) .
$$

This is a weak homotopy equivalence because $M$ is a manifold and $f$ is cellular. Then, it follows from Theorem 1.3 that the $\operatorname{map} F_{n}(f) \rightarrow F_{n-1,1}(f, M)$ is also a weak homotopy equivalence and the result follows inductively.

The $\operatorname{map} f_{*}: F_{n}(f) \rightarrow F_{n}(Y)$ is again proper and cell-like/cellular, and therefore a (proper) homotopy equivalence, see [8, Proposition 4.4].

REMARK 3.2. In the case where $Y$ is also a topological $d$-manifold, Proposition 3.1 specialises to a different proof of [8, Theorem 4.5]. The statement in [8] only requires that $f$ is proper and cell-like, but cell-like maps in this case are indeed cellular. This is a consequence of the cellularity criterion of McMillan [6] for dimension $d \neq 4$, and Repovš [9] for $d=4$ (see also Lacher [4, Theorem 4.3]). In dimension 3, the cellularity of proper cell-like maps requires also the proof of the Poincare conjecture. See [7] for a nice survey. In general, a proper, cell-like map $f: M \rightarrow Y$ where $M$ is a topological manifold is called a cell-like resolution of $Y$. Cell-like resolutions play a crucial role in the recognition problem for topological manifolds.

3.2. Abstract transversality. We comment on the abstract transversality context of [1] and in particular, the results of [1, Theorem 3.7] and [3, Corollary 2.9]. First, as a consequence of Theorem 1.3, we have the following proposition.

Proposition 3.3. Let $B$ be a space, $X_{\bullet}$ a simplicial space, and $p_{\bullet}: X_{\bullet} \rightarrow B a$ simplicial map which is a degreewise Serre fibration ( $B$ is regarded as a constant simplicial space). Let $U_{\bullet} \subseteq X_{\bullet}$ be a degreewise open subspace. Suppose that for all $k \geq 0$ and $b \in B$, the map

$$
U_{k} \cap p_{k}^{-1}(b) \rightarrow p_{k}^{-1}(b)
$$

is $(n+1-k)$-connected. Then, the map $\left\|U_{\bullet}\right\| \rightarrow\left\|X_{\bullet}\right\|$ is $(n+1)$-connected. (Here, $\|\cdot\|$ denotes the fat realization of the simplicial space.) 
Proof. By Theorem 1.3, the map $U_{k} \rightarrow X_{k}$ is $(n+1-k)$-connected. Then, it follows from well-known results about the fat realization (see, e.g., [3, Proposition 2.7]) that $\left\|U_{\bullet}\right\| \rightarrow\left\|X_{\bullet}\right\|$ is $(n+1)$-connected. Note that the fat realization is the same as the geometric realization of the underlying semi-simplicial space.

The abstract trasversality context concerns a setup similar to Proposition 3.3 but with more special and weaker assumptions. The setup involves a space $B$, a simplicial space $F_{\bullet}$, and an open subspace $U_{\bullet} \subseteq B \times F_{\bullet}$. The fibre $F_{\bullet}$ at $b \in B$ of the projection

$$
B \times F_{\bullet} \rightarrow B
$$

is regarded as the space of potential structures on $b$. The subspace $U_{\text {. consists of }}$ those structures which are admissible or transverse. The question is whether a fibrewise transversality statement, that is, a statement that the map

$$
\left\|U_{b, \bullet}\right\|:=\left\|U_{\bullet} \cap\left(\{b\} \times F_{\bullet}\right)\right\| \rightarrow\left\|F_{\bullet}\right\|
$$

is a weak equivalence for all $b \in B$, implies a global transversality statement saying that

$$
\left\|U_{\bullet}\right\| \rightarrow\left\|B \times F_{\bullet}\right\|
$$

is a weak equivalence. For concrete geometric examples of such questions, see [1] and [3]. Proposition 3.3 fails to fit directly in this context because it requires that the weak (or $n$-)equivalences are degreewise. The following proposition is a slight improvement of related results in [1] and [3] and concerns the case where the maps become fibrewise $n$-equivalences after geometric realization.

Proposition 3.4. Let $F_{\bullet}$ be a simplicial set and $B$ a Hausdorff space. Let $p_{\bullet}$ : $B \times F_{\bullet} \rightarrow B$ be the projection and $U_{\bullet} \subseteq B \times F_{\bullet}$ a degreewise open simplicial subspace. If

$$
\left\|U_{b, \bullet}\right\| \rightarrow\left\|F_{\bullet}\right\|
$$

is an $(n+1)$-equivalence for all $b \in B$, then so is $\left\|U_{\bullet}\right\| \rightarrow\left\|B \times F_{\bullet}\right\|$.

Proof. The map $\left\|U_{\bullet}\right\| \rightarrow B$ is a Serre microfibration [3, Proposition 2.8]. This uses that $B$ is Hausdorff. Note that the fibre of this map at $b \in B$ is $\left\|U_{b, \bullet}\right\|$ (see [3, Corollary 2.9]). Then, the result follows from Theorem 1.3.

REMARK 3.5. Special cases of this appear in [3] and [1]. The authors of [3] considered the case where $\left\|U_{b, \bullet}\right\|$ are $n$-connected, see [3, Corollary 2.9]. On the other hand, in [1], it was shown under different point-set topological assumptions that if the maps $\left\|U_{b, \bullet}\right\| \rightarrow\left\|F_{\bullet}\right\|$ are weak equivalences, then so is the map $\left\|U_{\bullet}\right\| \rightarrow\left\|B \times F_{\bullet}\right\|$, cf. $[1,3.6,3.7]$.

\section{REFERENCES}

1. M. Bökstedt and Ib Madsen, The cobordism category and Waldhausen's K-theory, in An alpine expedition through algebraic topology, Contemporary Mathematics 617, (Amer. Math. Soc., Providence, RI, 2014), 39-80.

2. C. O. Christenson and R. P. Osborne, Pointlike subsets of a manifold, Pacific J. Math. 24 (1968), 431-435. 
3. S. Galatius and O. Randal-Williams, Homological stability for moduli spaces of high dimensional manifolds. I, arXiv:1403.2334.

4. R. C. Lacher, Cell-like mappings. I, Pacific J. Math. 30 (1969), 717-731.

5. R. C. Lacher, Cell-like spaces, Proc. Amer. Math. Soc. 20 (1969), 598-602.

6. D. R. McMillan, Jr., A criterion for cellularity in a manifold, Ann. of Math. 79(2) (1964), 327-337.

7. W. J. R. Mitchell and D. Repovš, The topology of cell-like mappings, in Conference on Differential Geometry and Topology (Sardinia, 1988), Rend. Sem. Fac. Sci. Univ. Cagliari 58 (1988), 265-300.

8. G. Raptis, Some remarks on configuration spaces, Proc. Amer. Math. Soc. 139(5) (2011), 1879-1887.

9. D. Repovš, A criterion for cellularity in a topological 4-manifold, Proc. Amer. Math. Soc. 100(3) (1987), 564-566.

10. M. Weiss, What does the classifying space of a category classify?, Homology Homotopy Appl. 7(1) (2005), 185-195. 